\title{
The Effectiveness of Land and Water Based Resistance Training on Shoulder Rotator Cuff Strength and Balance of Youth Swimmers
}

\author{
by \\ Nuno Batalha, ${ }^{1,2}$, Sónia Dias ${ }^{1}$, Daniel A. Marinho ${ }^{2,3}$, José A. Parraca $^{1,2}$
}

The continuous execution of swimming techniques, supported mainly by the upper limbs, may cause shoulder rotator muscle imbalances, which leads to injuries. The aim of this study was to evaluate and compare the effects of two training programs on strength, balance and endurance of shoulder rotator cuff muscles in youth swimmers. Twentyfive male swimmers were evaluated and randomly divided into two groups - the land group $(n=13)$, which conducted a conventional dry-land training program with elastic bands, and the water group $(n=12)$, which conducted a water resistance program. In both groups, the level of strength of the shoulder rotators was evaluated with an isokinetic dynamometer on two occasions (baseline and after 10 weeks) using two protocols: i) three repetitions at $60 \%$ s; ii) twenty repetitions at $180 \%$ s. The land group significantly increased the unilateral ratios compared to the water group. The land group also decreased the external rotator levels of muscular fatigue. The dry-land training program conducted proved to be more effective than the one conducted in the water, allowing to reduce the muscle imbalance and to decrease muscle fatigue.

Key words: swimming, sports injuries, shoulder rotators; isokinetic strength.

\section{Introduction}

An agonistic-antagonistic muscle balance is key in all sports skills, providing joint stability and mobility, particularly in the upper body of overhead athletes (Bak and Magnusson, 1997; Maszczyk, et al. 2018). Maglischo (2003) describes swimming as an endurance sport, where a considerable training volume is needed to the achievement of certain goals, putting the shoulder joint in overload. Being a cyclical and bilateral sport, in which the propulsive forces are mainly produced by the upper limbs, through arm adduction and shoulder internal rotation, there is a high probability for shoulder injuries to occur (Blanch, 2004; Wanivenhaus et al., 2012; Stanula et al., 2012; Ostrowski et al., 2012).
The shoulder joint complex is characterized by a high degree of mobility, therefore, injury prevention programs for swimmers may require significant dynamic stabilization. These programs should focus on external rotation compensatory strengthening as well as on all joint dynamic and static muscle group stabilizers. However, due to the importance of shoulder joint dynamic stabilizers, absolute strength should not be considered equally relevant as the shoulder rotator muscle balance (Johnson et al., 2003). The usage of an isokinetic evaluation in the shoulder joint is common, allowing the access to values for both agonistic and antagonistic muscles recruited during

\footnotetext{
1 - Departamento de Desporto e Saúde, Escola de Ciência e Tecnologia, Universidade de Évora, Évora, Portugal.

2 - Research Centre of Sports, Health and Human Development, CIDESD, STRONG Research Community. University of Évora, Évora, Portugal.

3 - University of Beira Interior. Covilhã, Portugal.
} 
shoulder rotation (Ellenbecker and Davies, 2000). Unilateral ratios are commonly used to characterize the strength relationship between muscle groups (Cingel et al., 2007). These ratios, according to Ellenbecker and Roetert (2003), represent the quality of muscular balance, providing one of the key variables for diagnosing muscular balance/imbalance in any joint complex. The shoulder unilateral external rotation/internal rotation ratio (ER/IR ratio) is an important indicator when evaluating and interpreting the muscular function of the upper limbs (Ellenbecker and Davies, 2000).

The most frequent injury in swimming is the one of the shoulder joint complex, with a prevalence from 40 to $91 \%$ (Wanivenhaus et al., 2012). It is known that exclusive water training causes muscle imbalances between the internal rotators (IR) and external rotators (ER) (Batalha et al., 2013). Moreover, there are preventive means of injuries with traditional compensatory strength training programs (dry-land) for the shoulder rotators (Reinold et al., 2004; Swanik et al., 2002). However, to the best of our knowledge, there are no references to studies with specific compensatory strength training programs involving water exercises. One should be aware that, due to water density, aquatic movements have high resistance, with drag values of approximately 800 times higher than in the same movement executed on land. Hence, these resistive characteristics may be used for training programs in-water, especially when they allow the usage of body segment movements with a larger cross section area. Thus, the aim of this study was to evaluate and compare the effects of two training programs (dry-land and water exercises). It was hypothesized that water exercises would be effective in improving strength, balance and endurance of the shoulder rotators.

\section{Methods}

\section{Participants}

Twenty-five young male swimmers were included in the study. After the initial evaluation, they were randomly divided into two groups (Table 1): the water group (WG) $(n=12)$ and the land group (LG) $(n=13)$. The subjects needed to meet the following inclusion criteria: i) swimmers aged between 12 and 15 years old; ii) never having performed a shoulder rotator strength training program; iii) undergoing a minimum of 8 $\mathrm{h}$ of training per week; and iv) not having a clinical history of upper limb disorders (especially affecting the shoulders).

The overall objectives of the study were described to all participants. Both subjects and their legal guardians gave their written consent to participate in the study. The Ethics Committee of the seeding Institution (proceeding GD/33839/2013) gave their approval on all the procedures, which were under the Helsinki Declaration of 1975.

Each subject visited the laboratory one week before baseline testing to familiarize with the equipment and procedures to be used. One week after the familiarization, baseline evaluations were performed. The evaluations were carried out twice: at the beginning of the competitive season and after 10 weeks of intervention.

In order to verify whether there were differences in maturational stages of the two groups, the percentage of predicted mature height, based on the Khamis and Roche (1994) method, was measured in all evaluation periods. This method uses body height, body mass and mid-parent stature.

\section{Design and Procedures}

Isokinetic evaluation

The IR and ER isokinetic strength values were obtained through concentric contractions carried out on an isokinetic dynamometer (Biodex System 3 - Biodex Corp., Shirley, NY, USA). The subjects were seated and stabilized using Velcro tapes, avoiding upper body compensatory movements. The arms were in the scapular plane at 90 degrees of abduction, 90 degrees of elbow flexion, and the range of motion of 0 to 90 degrees as proposed elsewhere (Julienne et al., 2007; Tyler et al., 2005). The subjects' positioning and the segments' alignment were performed according to the instructions of the isokinetic dynamometer manufacturer (Biodex Medical Systems, 2000). Detailed explanation of the testing procedure was given to all participants before the onset of testing, with an emphasis on having each subject exert maximal effort within their tolerance level. After 15 min of a standardized warm up, the peak torque was evaluated during the performance of 3 repetitions at $60^{\circ} / \mathrm{s}$ and 20 repetitions at $180^{\circ} / \mathrm{s}$, 
with a one-minute rest period in between. Two trial repetitions at each speed were performed to prepare each subject for the test procedure. Standardized verbal instructions and encouragement were given to all subjects. The range of motion (ROM) halts were performed in accordance with the manufacturer's recommendations, in order to ensure that identical ROM were tested bilaterally and during follow-up. Protocols and evaluations were all corrected for the gravity effect.

The peak torque (PT), defined as the maximum torque produced by the shoulder at any point of the range of motion, was chosen to characterize the strength values of shoulder rotator muscles (Perrin, 1993). To analyze muscle balance, the ER/IR ratio [(ER/IR) x100] was calculated (Cingel et al., 2007). The endurance ratio calculation was performed according to the manufacturer's recommendations (Biodex Medical Systems, 2000), considering the mechanical work of the first third (W1) and the last third (W2) of 20 repetitions - [(W1W2)/W1]x100. This variable, being an indicator of muscle fatigue, was only used considering the protocol with 20 repetitions at $180^{\circ} / \mathrm{s}$.

Training program

The LG, along with aquatic training, completed a traditional dry-land strength training program during 10 weeks. Subjects used TheraBand ${ }^{\circledR}$ elastic resistance bands. The training program was held three times a week and consisted of three different exercises (Figure 1): Exercise One: the subjects initiated the exercise in an anatomical reference position with slightly higher upper limb abduction and with the elastic band in tension. Following this initial position, an additional upper limb abduction and external rotation was required, until an angle of 50 to 60 degrees between the arms and the torso was achieved; Exercise Two: the initial position was identical to the position described for Exercise One, progressing to simultaneous upper limb abduction at the scapular plane until the end of the range of motion, close to 160 degrees of abduction; Exercise Three: subjects adopted an initial standing position, with the elastic band anchored to a surface at the level between the waist and the shoulder. The arm was at $90^{\circ}$ elbow flexion and abduction, and $90^{\circ}$ shoulder flexion, progressing through an ER.
Before determining the initial training load, the groups performed two sessions in order to adapt to materials and training techniques. The initial load was implemented using red elastic bands. All swimmers performed 3 sets of each exercise described above with $30 \mathrm{~s}$ rest intervals. The first two sets involved 20 repetitions each, while the last set was performed until exhaustion. The training load progression was ensured since, during the last set, when swimmers reached 30 repetitions, the load intensity was increased, using an elastic band indicating the next level of color/resistance. This procedure was conducted independently for each exercise in the program. For all exercises, the execution technique was controlled by the coach in all sessions and the adaptation period. It was also requested that the execution speed was moderate.

The WG, together with usual water training, performed a specific compensatory water strength training program, which included three exercises executed three times a week (Figure 2): Exercise One: the subjects initiated this exercise with the forearm in a semi-pronated position, elbow at $90^{\circ}$ of flexion, arms close to the body, progressing to shoulder external rotation. While returning to the initial position, they internally rotated the arm and the forearm progressed to a pronated position, keeping the arms and elbows close to the body. During the exercise, they used a Thera-Band® red elastic band placed around the wrists; Exercise Two: very similar to the previous exercise, but instead of using the bands, the swim hand paddles were used. The hand paddles had a $116 \mathrm{~cm}^{2}$ area and were used differently; the back of the swim paddle was used to provide higher resistance in the movement of ER. In the returning movement to the initial position, in order not to induce resistance to the water, the swimmers turned the palm of the hand to the bottom (performing internal rotation without resistance); Exercise Three: in a seated position, the athletes performed sculling, favoring the external rotation in which the hand was placed in such a way that the palm offered the greatest possible resistance to the water, but in the internal rotation (returning to the initial position) the palm of the hand was turned to the bottom, thus eliminating resistance to the water, and recovered in a "passive" way.

The training load progression was 
promoted every two weeks: Week $1-3 \times 30$ s; Week $3-4$ x 30 s; Week $5-3$ x 45 s; Week $7-4$ x $45 \mathrm{~s}$; Week $9-5 \times 30 \mathrm{~s}$. The athletes had a $10 \mathrm{~s}$ pause between sets and 2 min between exercises. All exercises were performed exclusively with the upper limbs, without support in the swimming pool.

\section{Statistical analysis}

The Kolmogorov-Smirnov normality test with Lilliefors correction was initially used. Differences in baseline characteristics between groups were tested using the Student's $t$-test for independent samples. The training effects within and between groups were evaluated using analyses of variance for repeated measures, adjusted to the baseline and maturation values used as covariates (ANCOVA), with Bonferroni post-hoc tests. Effect sizes are reported as partial eta-squared $\left(\eta_{\mathrm{p}}{ }^{2}\right)$, with cut-off values of $0.01,0.06$, and 0.14 for small, medium, and large effects, respectively (Cohen, 1988).

In addition to the $p$ values, we provided detailed statistics, including the mean and 95\% confidence intervals, to better depict the changes between evaluation periods within groups. The change to 10 weeks for a given group was defined as an increase or a decrease from baseline to 10 weeks for that group. All the analyses were performed with SPSS (version 20.0; SPSS Inc, Chicago, IL), and the level of significance was set at $p \leq 0.05$ for all tests.

\section{Results}

The overall characteristics of both groups were similar in terms of age, body mass, training per week, and maturational status. Table 2, regarding the assessment made at $60^{\circ} / \mathrm{s}$, shows significant differences in the dominant shoulder between the two training programs from baseline to 10 weeks in IR strength and unilateral ratios ( $p$ $=0.028$; and $p=0.015$, respectively), also with large effect sizes $\left(\eta_{\mathrm{p}}{ }^{2}>0.14\right)$. In these cases, the land-based training program appeared to be more effective. Regarding the non-dominant shoulder results, the same trend was observed as the landbased training program was also more effective in increasing IR strength $\left(p=0.013 ; \eta_{\mathrm{p}}{ }^{2}=0.221\right)$ and the ER/IR ratio $\left(p=0.016 ; \eta_{p^{2}}=0.181\right)$.

Regarding the evaluation carried out at $180^{\circ}$ /s (Table 3), the results are similar to the ones previously described. In both shoulders, betweengroup differences were found from baseline to 10 weeks in IR strength $\left(p=0.023 ; \eta_{\mathrm{p}}{ }^{2}=0.147\right.$ in the dominant shoulder; $p=0.036 ; \eta_{p}{ }^{2}=0.167$ in the non-dominant shoulder) and ER/IR ratios ( $p=$ 0.007; $\eta_{\mathrm{p}^{2}}=0.222$ in the dominant shoulder; $p=$ $0.017 ; \eta_{p^{2}}=0.194$ in the non-dominant shoulder). Once again, the land-based program appeared to be more effective, not only in increasing IR strength, but also in the shoulder rotators balance.

With respect to endurance ratios (Figure 3), between-group differences were found in the ER with large effect sizes, only in the dominant shoulder $\left(p=0.023 ; \eta_{p}^{2}=0.168\right)$. Within-group differences (a significant decrease) were found in the ER of the land experimental group in both shoulders.

\section{Table 1}

Baseline characteristics of the subjects

\begin{tabular}{|c|c|c|c|}
\hline & Water Group $(\mathrm{n}=12)$ & Land Group $(n=13)$ & $p$ \\
\hline Age (y) & $13.28 \pm 0.96$ & $13.52 \pm 0.92$ & 0.687 \\
\hline Body Mass (kg) & $49.30 \pm 7.90$ & $48.59 \pm 6.51$ & 0.993 \\
\hline Body Height (cm) & $159.61 \pm 5.62$ & $162.59 \pm 9.46$ & 0.389 \\
\hline Training per week (sessions) & $5.70 \pm 0.67$ & $6.09 \pm 0.30$ & 0.216 \\
\hline$\%$ of predicted mature height & $92.31 \pm 3.83$ & $91.97 \pm 2.47$ & 0.745 \\
\hline
\end{tabular}

Values are expressed as mean \pm SD; $p$ values of student's t-test for independent samples 
Table 2

Comparative training effects on the Peak-torques (Nm) of Internal Rotators (IR), External Rotators (ER) and ER/IR ratios (\%) of the dominant and non-dominant shoulder at $60 \%$ s.

\section{Dominant Shoulder $-60 \%$}

Baseline Changes at 10 Weeks

$($ mean \pm SD $)$ Mean $(95 \% \mathrm{CI})$ p ES

PT - ER

\begin{tabular}{lllll}
\hline Water Group & $18.11 \pm 4.98$ & $-1.21(-4.13$ to 1.71$)$ & 0.237 & 0.080
\end{tabular}

\section{PT - IR}

\begin{tabular}{lllll}
\hline Water Group & $23.12 \pm 7.01^{\mathrm{a}}$ & $7.03(1.20 \text { to } 12.86)^{*}$ & 0.028 & 0.157 \\
\cline { 1 - 3 } Land Group & $31.72 \pm 8.04^{\mathrm{a}}$ & $-2.10(-7.66$ to 3.46$)$ & \\
\hline ER/IR Ratio & & & \\
\hline Water Group & $79.51 \pm 7.80^{\mathrm{a}}$ & $-20.63(-35.81 \text { to }-5.45)^{*}$ & 0.015 & 0.241 \\
\hline Land Group & $63.96 \pm 22.08^{\mathrm{a}}$ & $6.32(-8.16$ to 20.80$)$ & & \\
\hline
\end{tabular}

Non-Dominant Shoulder $-60 \%$

$\begin{array}{lllll}\begin{array}{l}\text { Baseline } \\ (\text { mean } \pm \mathrm{SD})\end{array} & \text { Changes at } 10 \text { Weeks } & & \\ & \text { Mean }(95 \% \mathrm{CI}) & p & \text { ES }\end{array}$

PT - ER

\begin{tabular}{lllll}
\hline Water Group & $16.34 \pm 5.02$ & $0.27(-2.42$ to 2.96$)$ & 0.689 & 0.028
\end{tabular}

PT - IR

\begin{tabular}{llll}
\hline Water Group & $20.95 \pm 6.64^{\mathrm{a}}$ & $6.36(1.42 \text { to } 11.30)^{*}$ & 0.013
\end{tabular}$\quad 0.221$

ER/IR Ratio

\begin{tabular}{lllll}
\hline Water Group & $78.80 \pm 11.88^{\mathrm{a}}$ & $-15.79(-29.63 \text { to }-1.95)^{*}$ & \\
\cline { 1 - 2 } Land Group & $62.43 \pm 15.73^{\mathrm{a}}$ & $8.38(-4.81$ to 21.58$)$ & 0.181
\end{tabular}

$P T=$ Peak-Torque $E R=$ External Rotators; $I R=$ Internal Rotators $p$ values for differences between groups after 10 weeks; ES - Effect size

${ }^{a} p<0.05$ on baseline outcome between Groups

* Within-group differences after 10 weeks. 
Table 3

Comparative training effects on Peak-torques (Nm) of Internal Rotators (IR),

External Rotators (ER) and ER/IR ratios (\%) of the dominant and non-dominant shoulder at 180\%/s.

Dominant Shoulder $-180 \%$

\begin{tabular}{|c|c|c|c|c|}
\hline & $\begin{array}{l}\text { Baseline } \\
(\text { mean } \pm S D)\end{array}$ & $\begin{array}{l}\text { Changes at } 10 \text { Weeks } \\
\text { Mean }(95 \% \mathrm{CI})\end{array}$ & $p$ & ES \\
\hline \multicolumn{5}{|l|}{ PT - ER } \\
\hline Water Group & $17.67 \pm 4.73$ & $1.60(-.97$ to 4.17$)$ & \multirow{2}{*}{0.150} & \multirow{2}{*}{0.039} \\
\hline Land Group & $16.44 \pm 4.76$ & $4.15(1.69$ to 6.60$) *$ & & \\
\hline \multicolumn{5}{|l|}{ PT - IR } \\
\hline Water Group & $23.91 \pm 5.41$ & $8.59(3.31 \text { to } 13.87)^{*}$ & \multirow{2}{*}{0.023} & \multirow{2}{*}{0.147} \\
\hline Land Group & $28.12 \pm 7.75$ & $-.018(-5.05$ to 5.02$)$ & & \\
\hline \multicolumn{5}{|l|}{ ER/IR Ratio } \\
\hline Water Group & $73.69 \pm 5.69$ & $-8.95(-18.65$ to .75$)$ & \multirow{2}{*}{0.007} & \multirow{2}{*}{0.222} \\
\hline Land Group & $62.00 \pm 20.54$ & $11.06(1.84 \text { to } 0.28)^{*}$ & & \\
\hline \multicolumn{5}{|c|}{ Non-Dominant Shoulder - $180 \% / s$} \\
\hline & $\begin{array}{l}\text { Baseline } \\
(\text { mean } \pm \mathrm{SD})\end{array}$ & $\begin{array}{l}\text { Changes at } 10 \text { Weeks } \\
\text { Mean }(95 \% \mathrm{CI})\end{array}$ & $p$ & ES \\
\hline \multicolumn{5}{|l|}{ PT - ER } \\
\hline Water Group & $16.84 \pm 4.24$ & $1.69(-1.12$ to 4.50$)$ & \multirow{2}{*}{0.657} & \multirow{2}{*}{0.021} \\
\hline Land Group & $17.55 \pm 5.85$ & $2.53(-.15$ to 5.21$)$ & & \\
\hline \multicolumn{5}{|l|}{ PT - IR } \\
\hline Water Group & $21.83 \pm 5.47^{a}$ & $7.45(2.46 \text { to } 12.44)^{*}$ & \multirow{2}{*}{0.036} & \multirow{2}{*}{0.167} \\
\hline Land Group & $28.40 \pm 7.49^{a}$ & $.009(-4.75$ to 4.77$)$ & & \\
\hline
\end{tabular}

ER/IR Ratio

\begin{tabular}{lllll}
\hline Water Group & $77.72 \pm 8.85^{\mathrm{a}}$ & $-10.08(-22.77$ to 2.61$)$ & 0.017 & 0.194 \\
\cline { 1 - 3 } Land Group & $62.43 \pm 14.73^{\mathrm{a}}$ & $11.86(-.24$ to 23.97$)$ & & \\
\hline
\end{tabular}

$P T=$ Peak-Torque; ER = External Rotators; IR = Internal Rotators

$p$ values for differences between groups after 10 weeks; ES - Effect size

a $p<0.05$ on baseline outcome between Groups

* Within-group differences after 10 weeks. 


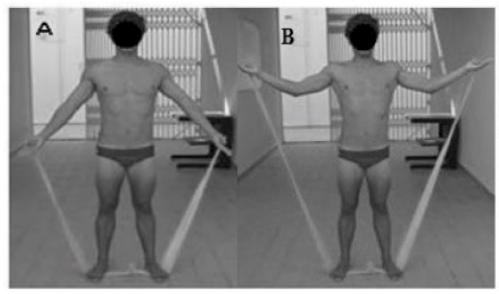

Exercise 1

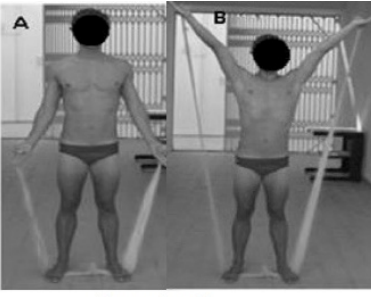

Exercise 2

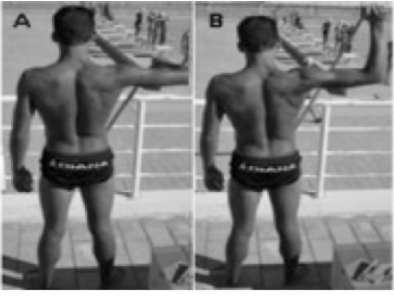

Exercise 3

Figure 1

Exercises of the Land Group strength-training programme.

(a) Initial position; (b) final position

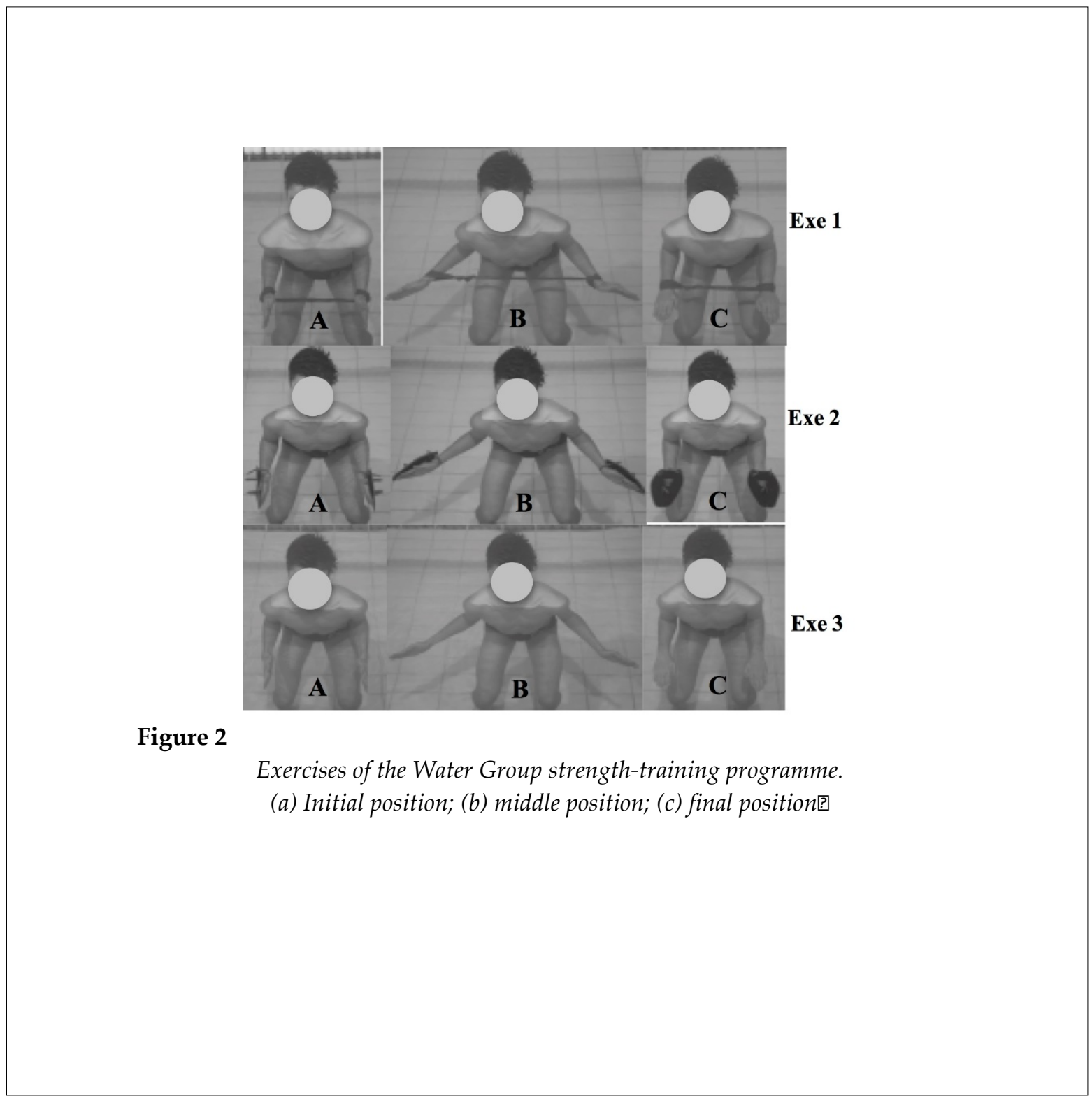




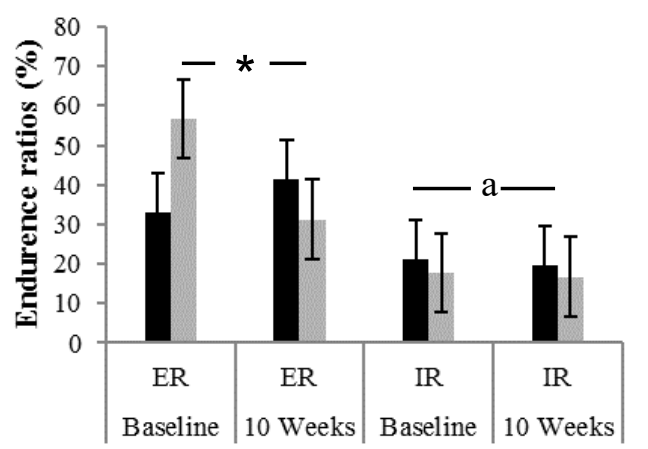

A

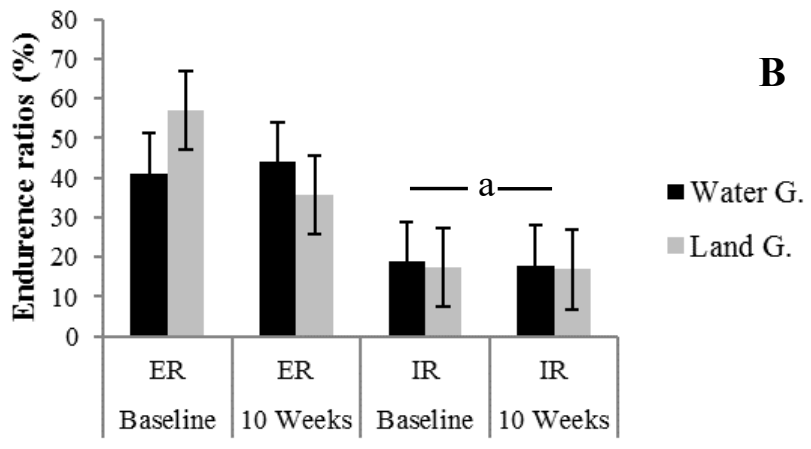

Figure 3

Endurance ratios for the dominant $(A)$ and non-dominant $(B)$ shoulder $a-W i t h i n-L a n d$ group differences between baseline and 10 weeks;

* - differences between groups ( $p \leq 0.05)$

$E R$ - External rotation; IR - Internal rotation

\section{Discussion}

The aim of this study was to evaluate and compare the effects of two different training programs on strength, balance and endurance of the shoulder rotators in youth swimmers. Despite the fact that we had no control group, our results confirm that the land-based program was more effective in increasing the shoulder rotators balance and reducing the endurance ratios.

Regarding the ER and IR strength training effects (Tables 2 and 3), the results are very similar for both protocols. In all the assessments and in both limbs (dominant and non-dominant), there were significant within-group increases for IR strength from the beginning to 10 weeks in the WG. These results match previous swimmer's data (Batalha et al., 2013; Ramsi et al., 2004;) in which there were also significant increases in IR strength values throughout a training macrocycle (Batalha et al., 2015) and a competitive season (Batalha et al., 2013). It is known that aquatic training increases IR strength in a disproportionate way, when compared to their antagonists (Kluemper and Hazelrigg, 2006; 
Ramsi et al., 2004). According to the literature, explanations are based on two main findings: on the one hand, some studies support these results on a biomechanical analysis of swimming techniques basis; evidence suggests that the internal rotators are more intensely stimulated due to the repeated concentric contractions to which they are subjected during the propulsive phases of swimming (Johnson et al., 2003; Yanai and Hay, 2000); on the other hand, O'Donnell et al. (2005) claimed that swimming techniques promote muscle imbalances that cause stress of the glenohumeral capsular ligament complex, contributing to instability of the shoulder joint, with obvious consequences on the ability to produce strength. However, in the mentioned studies, the swimmers performed only water training and there were no compensatory strength training exercises as in the WG in the present study.

Also regarding the IR strength values, we found significant differences with large effect sizes between groups in the assessments in both protocols and shoulders. In fact, the WG significantly increased the IR strength values comparing to the LG. If we consider that the aquatic training was similar for both groups and that the maturational levels are were identical, we cannot find an obvious explanation for the fact that the WG raised IR strength to a higher level compared to the LG. One can assume that probably the LG, having higher ER training effects, might have changed in some way the "normal" pattern of neuromuscular function and consequently the intramuscular coordination, compared to the WG. However, complementary studies should be carried out to clarify this situation, namely recording shoulder rotators electromyography activity before and after compensatory strength training.

With regard to muscular balance, translated by the ER/IR ratios, the most notable results are a consequence of the previously mentioned strength value increases. Invariably, in all the assessments and on both shoulders, there were differences between groups by the end of the 10th week. The LG always increased the ER/IR ratios, although only with within-group differences on the dominant shoulder at $180^{\circ} / \mathrm{s}$. As far as the WG was concerned, results were totally opposite, as in all assessments the ratio values decreased, with differences in both shoulders at $60^{\circ} / \mathrm{s}$. Since the unilateral ratios characterize the quality of muscular balance (Ellenbecker and Roetert, 2003), and no differences were found between the maturity levels of both groups, we can conclude that the dry-land strength training program was more effective than the water training program, in increasing the shoulder rotators muscular balance.

Regarding the LG results of both isokinetic strength protocols, our findings agree with previous research, which included compensatory training programs focusing on the shoulder rotator muscles (Kluemper and Hazelrigg, 2006; Malliou et al., 2004; Swanik et al., 2002). Significant gains in strength were recorded for recruited muscle groups in the exercises. In some studies (Beneka et al., 2002; Malliou et al., 2004; Swanik et al., 2002) concerning unilateral ratios, the findings contrasted with those presented in the current study, with diminishing ratios after interventions. The main difference lies in the type of strength training employed, which did not seem to relate to the ER. Therefore, IR strength values were significantly higher when compared to their antagonists. This fact, together with our results, provides strong support for the use of compensatory land-based strength training on the swimmer's shoulder rotators, which should include both ER strengthening as well as shoulder stabilization.

Regarding the unilateral ratios in the WG, we have no other data to compare. However, there are previous research findings in swimming, tennis, water polo and badminton that point to normative ER/IR ratio values of 66 to $75 \%$ (Cingel et al., 2007; Ellenbecker and Roetert, 2003; Ramsi et al., 2004). The decrease in these values, as occurred in the water group, has been linked to instability and imbalance in the glenohumeral joint (Leroux et al., 1994). Considering these normative values, our results, in both protocols, registered some values below $66 \%$. This means, according to Leroux et al. (1994), that they should therefore be considered negative, as they are within the range associated with muscular imbalance. Byram et al. (2010), in a longitudinal study, reinforce the idea that low ratios are correlated to future injuries. In this way, the water-training program of the present study did not show benefits for the shoulder rotator 
muscular balance in youth swimmers.

The endurance ratio results challenge the assumption that water training improves the shoulder rotator muscle endurance of competitive swimmers. The intragroup analysis of the LG showed a significant increase in endurance only in the ER on both shoulders. We also found differences between groups, with corresponding large effect sizes, in the ER endurance ratios of the dominant shoulder, with better results in the LG. Thus, dry-land strength training proved to be more effective also in the decrease of the ER muscle fatigue. Considering that with increased endurance ratios competitive swimmers may be more likely to develop shoulder pain (Beach et al., 1992), it is important to consider dry-land strength training similar to the one presented in this study, to prevent shoulder injury.

The main limitation of this study was the fact that we did not have a control group, which suggests some caution in interpreting the results and conclusions. Another limitation is related to the isokinetic testing position. Swimmers were seated with a single arm elevated at the scapular plane, which is not a swimming-specific position. A prone testing position might be more suitable for swimmers; however, this position was not an option using this assessment tool. Finally, another limitation lies in the fact that there was no accurate assessment of the force produced in the different exercises in both groups.

\section{Conclusions}

The findings of the present study suggest that the prescribed dry-land shoulder strengthening exercises, when compared with the water training program, represent a more useful compensatory training option for youth competitive swimmers, since there is an increase in shoulder rotators balance and ER endurance. Therefore, swimming coaches should use mainly dry-land strength-training programs, focusing specifically on strengthening the ER and stabilizers of the shoulder joint.

\section{Acknowledgements}

This project was supported by the National Funds through FCT - Portuguese Foundation for Science and Technology (UID/DTP/04045/2013) - and the European Fund for regional development (FEDER) allocated by the European Union through the COMPETE 2020 Programme (POCI-01-0145-FEDER-006969).

\section{References}

Bak K, Magnusson SP. Shoulder strength and range of motion in symptomatic and pain-free elite swimmers. Am J Sports Med, 1997; 25: 454-459

Batalha N, Raimundo A, Tomas-Carus P, Barbosa T, Silva A. Shoulder Rotator Cuff Balance, Strength and Endurance in Young Swimmers During A Competitive Season. J Strength Cond Res, 2013; 27: 25622568

Batalha N, Marmeleira J, Garrido N, Silva AJ. Does a water-training macrocycle really create imbalances in swimmers' shoulder rotator muscles? Eur J Sport Sci, 2015; 15: 167-172

Beach ML, Whitney SL, Dickoff-Hoffman S. Relationship of shoulder flexibility, strength, and endurance to shoulder pain in competitive swimmers. J Orthop Sports Phys Ther, 1992; 16: 262-268

Beneka A, Malliou P, Giannakopoulos K, Kyrialanis P, Godolias G. Different training modes for the rotator cuff muscle group. A comparative study. Isokinet Exerc Sci, 2002; 10: 73-79

Biodex Medical Systems Inc. Biodex system 3 quick set - application/operation manual. Shirley: NewYork, 2000; 29-32. Available at: http://www.biodex.com/sites/default/files/830000man_04345.pdf

Blanch P. Conservative management of shoulder pain in swimming. Phys Ther Sport, 2004; 5: 109-124

Byram IR, Bushnell BD, Dugger K, Charron K, Harrell FE, Noonan TJ. Preseason shoulder strength measurements in professional baseball pitchers: identifying players at risk for injury. Am J Sports Med, 2010; 38: 1375-1382 
Cingel R, Kleinrensinkb G, Mulderc P, Bied R, Kuiperse H. Isokinetic strength values, conventional ratio and dynamic control ratio of shoulder rotator muscles in elite badminton players. Isokinet Exerc Sci, 2007; 15: 287-293

Cohen J. Statistical power analysis for the behavioral sciences (2nd ed.). Hillsdale, NJ: Lawrence Erlbaum; 1998

Drouin JM, Valovich TC, Shultz SJ, Gansneder BM, Perrin DH. Reliability and validity of the Biodex System 3 Pro Isokinetic Dynamometer velocity, torque and position measurements. Eur J Appl Physiol, 2004; 91: $22-29$

Ellenbecker TS, Davies GJ. The application of isokinetics in testing and rehabilitation of the shoulder complex. J Athl Train, 2000; 35: 338-350

Ellenbecker TS, Roetert EP. Age specific isokinetic glenohumeral internal and external rotation strength in elite junior tennis players. J Sci Med Sport, 2003; 6: 63-70

Gozlan G, Bensoussan L, Coudreuse JM, Fondarai J, Gremeaux V, Viton JM, Delarque A. Isokinetic dynamometer measurement of shoulder rotational strength in healthy elite athletes (swimming, volley-ball, tennis): Comparison between dominant and nondominant shoulder. Ann Readapt Med Phys, 2006; 49: 8-15

Johnson JN, Gauvin J, Fredericson M. Swimming Biomechanics and Injury Prevention. The Physician and Sports Medicine, 2003; 31: 41-48

Julienne R, Gauthier A, Moussay S, Davenne D. Isokinetic and electromyographic study of internal and external rotator muscles of tennis player. Isokinet Exerc Sci, 2007; 15: 173-183

Khamis HJ, Roche AF. Predicting adult stature without using skeletal age: the Khamis-Roche method. Pediatrics, 1994; 94: 504-507

Kluemper M, Hazelrigg H. Effect of stretching and strengthening shoulder muscles on forward shoulder posture in competitive swimmers. J Sport Rehabil, 2006; 15: 58-70

Leroux JL, Codine P, Thomas E, Pocholle M, Mailhe D, Blotman F. Isokinetic evaluation of rotational strength in normal shoulders and shoulders with impingement syndrome. Clin Orthop Relat Res, 1994; 304: 108-115

Maglischo EW. Swimming fasted. Champaign, Illinois: Human Kinetics Publishers, Inc; 2003

Malliou PC, Giannakopoulos K, Beneka AG, Gioftsidou A, Godolias G. Effective ways of restoring muscular imbalances of the rotator cuff muscle group: a comparative study of various training methods. $\mathrm{Br} \mathrm{J}$ Sports Med, 2004; 38: 766-772

Maszczyk A, Gołaś A, Pietraszewski P, Kowalczyk M, Cięszczyk P, Kochanowicz A, Smółka W, Zając A. Neurofeedback for the enhancement of dynamic balance of judokas. Biol. Sport, 2018; 35: 99-102

O'Donnell CJ, Bowen J, Fossati J. Identifying and managing shoulder pain in competitive swimmers. How to minimize training flaws and other risks. Phys Sportsmed, 2005; 33: 27-35

Ostrowski A, Strzała M, Stanula A, Juszkiewicz M, Pilch W, Maszczyk A. The Role of Training in the Development of Adaptive Mechanisms in Freedivers. J Hum Kinet, 2012; 32: 197-210

Perrin DH. Isokinetic Exercise and Assessment. Champaign, IL: Human Kinetics; 1993

Ramsi M, Swanik KA, Swanik C, Straub S, Maltacola C. Shoulder-Rotator Strength of High School Swimmers Over the Course of a Competitive Season. J Sport Rehabil, 2004; 13: 9-18

Reinold MM, Wilk KE, Fleisig GS, Zheng N, Barrentine SW, Chmielewski T, Cody RC, Jameson GG, Andrews JR. Electromyographic analysis of the rotator cuff and deltoid musculature during common shoulder external rotation exercises. J Orthop Sports Phys Ther, 2004; 34: 385-394

Stanula A, Maszczyk A, Roczniok R, Pietraszewski P, Ostrowski A, Zając A, Strzała M. The Development and Prediction of Athletic Performance in Freestyle Swimming. J Hum Kinet, 2012; 32: 97-107

Swanik KA, Swanik CB, Lephart SM, Huxel K. The effect of functional training on the incidence of shoulder pain and strength in intercollegiate swimmers. J Sport Rehabil, 2002; 2: 140-154 
Tyler TF, Nahow RC, Nicholas SJ, McHugh MP. Quantifying shoulder rotation weakness in patients with shoulder impingement. J Shoulder Elbow Surg, 2005; 14: 570-574

Wanivenhaus F, Fox AJ, Chaudhury S, Rodeo SA. Epidemiology of injuries and prevention strategies in competitive swimmers. Sports Health, 2012; 4: 246-251

Yanai T, Hay JG. Shoulder impingement in front- crawl swimming: II. Analysis of stroking technique. Med Sci Sports Exerc, 2000; 32: 30-40

\section{Corresponding author:}

\section{Nuno Batalha}

University of Évora. Sport and Health Department.

R. Reguengos de Monsaraz, no 14. 7000-727 Évora - Portugal.

Phone/Fax: (+351)962435000 - (+351)266769521

E-mail: nmpba@uevora.pt 